\title{
Multiwavelength determination of the density and total mass of the EUV filament observed by SoHO/CDS, SoHO/SUMER and MSDP/VTT
}

\author{
P. Schwartz ${ }^{1}$, B. Schmieder ${ }^{2}$, \\ P. Heinzel ${ }^{1}$ and U. Anzer ${ }^{3}$ \\ ${ }^{1}$ Astronomical Institute, Academy of Sciences of the Czech Republic, CZ-25165 Ondřejov, \\ Czech Republic email: schwartz@asu.cas.cz, pheinzel@asu.cas.cz \\ ${ }^{2}$ Observatoire de Paris, Section Meudon, LESIA, F-92195 Meudon Principal Cedex, France \\ email: brigitte.schmieder@obspm.fr \\ ${ }^{3}$ Max-Planck-Institut für Astrophysik, Karl-Schwarzschild-Strasse 1, D-85740 Garching, \\ Germany email: ula@mpa-garching.mpg.de
}

\begin{abstract}
It was found that filaments observed in EUV could be much more extended than in $\mathrm{H} \alpha$. These extended dark structures visible in EUV are named EUV filaments. Their parts seen only in EUV (not observable in $\mathrm{H} \alpha$ because of low opacity at the $\mathrm{H} \alpha$ wavelength) are called EUV-filament extensions (or simply EUV extensions). For the EUV filament observed by SoHO on 15 October 1999 as northern polar crown filament, only a few small dark structures were seen in $\mathrm{H} \alpha$. This suggests that the mass of the EUV extension is larger than, or at least comparable with, the mass of the parts of the filament observed in $\mathrm{H} \alpha$. In our previous work we determined the 3D structure of the EUV extension of this EUV filament. In this paper we present the determinations of mass and average density of this EUV extension. For better density estimates we interpret the hydrogen Lyman lines observed by SUMER using non-LTE radiative transfer code. From the best fitting of Lyman lines we obtained a reasonable model of the EUV extension with low gas pressure, temperatures from $2 \times 10^{4} \mathrm{~K}$ to $10^{5} \mathrm{~K}$ and with extended prominence-corona transition regions.
\end{abstract}

\section{Introduction}

It was found (Heinzel et al. 2001, Schmieder et al. 2003) that solar filaments observed in EUV lines are more extended than in $\mathrm{H} \alpha$. Parts of the filament visible only in EUV are invisible in $\mathrm{H} \alpha$ due to their very low opacity in this line. These parts were named 'EUV-filament extensions' or simply 'EUV extensions'. The manifestation of the EUV filament as dark structure is due to absorption at the EUV continuum by filament plasma and due to volume blocking (Heinzel et al. 2003a). The volume blocking mechanism is based on the fact that coronal lines cannot be emitted from the volume occupied by relatively cold EUV-filament plasma. The importances of volume blocking and absorption for the intensity depression are comparable (Schwartz et al. 2004). Schwartz et al. (2004) determined the 3D-structure of an EUV filament using the spectroscopic model of Heinzel et al. (2003a) and found that EUV filaments are comparable in height with their $\mathrm{H} \alpha$ counterparts. This result was confirmed by Schmieder et al. (2004) for another EUV filament where the heights were estimated also from on-disk projection (the filament was rather far from solar disk center). 


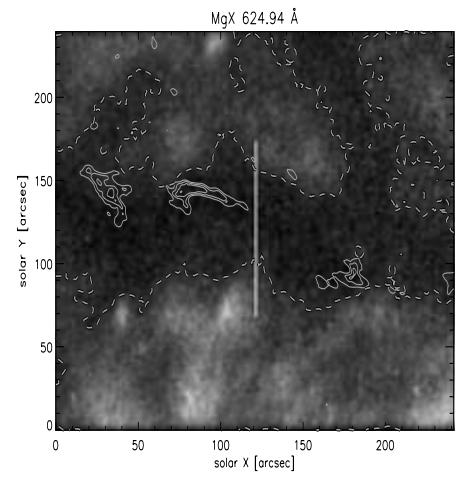

Figure 1. CDS observations of the $\mathrm{MgX} 624.94 \AA$ line. The dark area of the EUV filament is bordered by a dashed-line contour. The darkest areas seen in $\mathrm{H} \alpha$ are marked by full-line contours. The vertical white bar is the position of the SUMER slit.

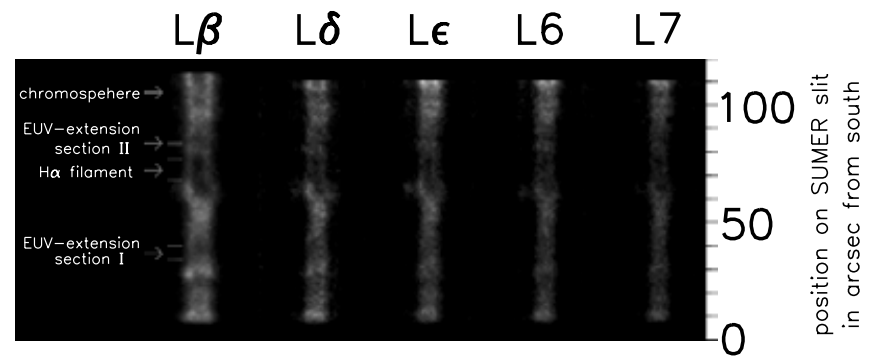

Figure 2. Hydrogen Lyman spectra observed by SoHO/SUMER. The ordinate is the position on the spectrograph slit from solar south. On the left we mark parts of the spectra belonging to the $\mathrm{H} \alpha$ filament with very deep self-absorption in the line center and to the chromosphere with only slight intensity depression in the line center. Also two sections belonging to the EUV extension are indicated. Average profiles from these two sections were used for modelling the properties of the EUV-extension plasma.

The EUV filament studied by Schwartz et al. (2004) was observed by SoHO/CDS (Coronal Diagnostic Spectrometer) (Harrison et al. 1995), SoHO/SUMER (Solar Ultraviolet Measurements of Emitted Radiation) (Wilhelm et al. 1995), both on SoHO (Solar and Heliospherical Observatory) and its $\mathrm{H} \alpha$ counterparts were observed by VTT/MSDP (Multichannel Subtractive Double Pass spectrograph on Vacuum Tower Telescope) on 15 October 1999 at position N37.6 E19.3 (northern polar crown filament). Only a few small $\mathrm{H} \alpha$ islands were observed inside of a wide EUV filament channel. In determination of 3D-structure of the EUV extension we used CDS observations of two coronal EUV lines (MgX 624.94 $\AA$ and SiXII $520.60 \AA$ ) with wavelengths between 504 and $912 \AA$ (i.e. between the HeI resonance continuum head and the hydrogen Lyman continuum head). Therefore the radiation of these lines is absorbed only by hydrogen plasma. The resulting heights of the EUV-filament extension are around $15000 \mathrm{~km}$ for the bottom and around $40000 \mathrm{~km}$ for the top boundaries although there are significant variations around these representative values.

In this work we present our determinations of an average plasma density and total mass of the EUV filament extensions, which are estimated by using the 3D structure previously determined from the spectroscopic model. Using the non-LTE radiative transfer code (Heinzel et al. 1997) for computing theoretical hydrogen line profiles we estimated average values of temperature and pressure of the EUV-extension plasma.

\section{Observations}

All observations were made on 15 October 1999 between 10:00 and 12:00 UT. For the 3D-structure determination (see Schwartz et al. 2004) we used $\mathrm{H} \alpha$ observations made by MSDP/VTT and the CDS observations of two coronal EUV lines: MgX $624.94 \AA$ (Figure 1) and SiXII 520.60 A. SUMER observations of the hydrogen Lyman lines L $\beta$, $\mathrm{L} \delta, \mathrm{L} \varepsilon, \mathrm{L} 6$ and L7 (Figure 2) were made with the spectrograph slit crossing the EUV filament in the center of the CDS raster (see Figure 1). 

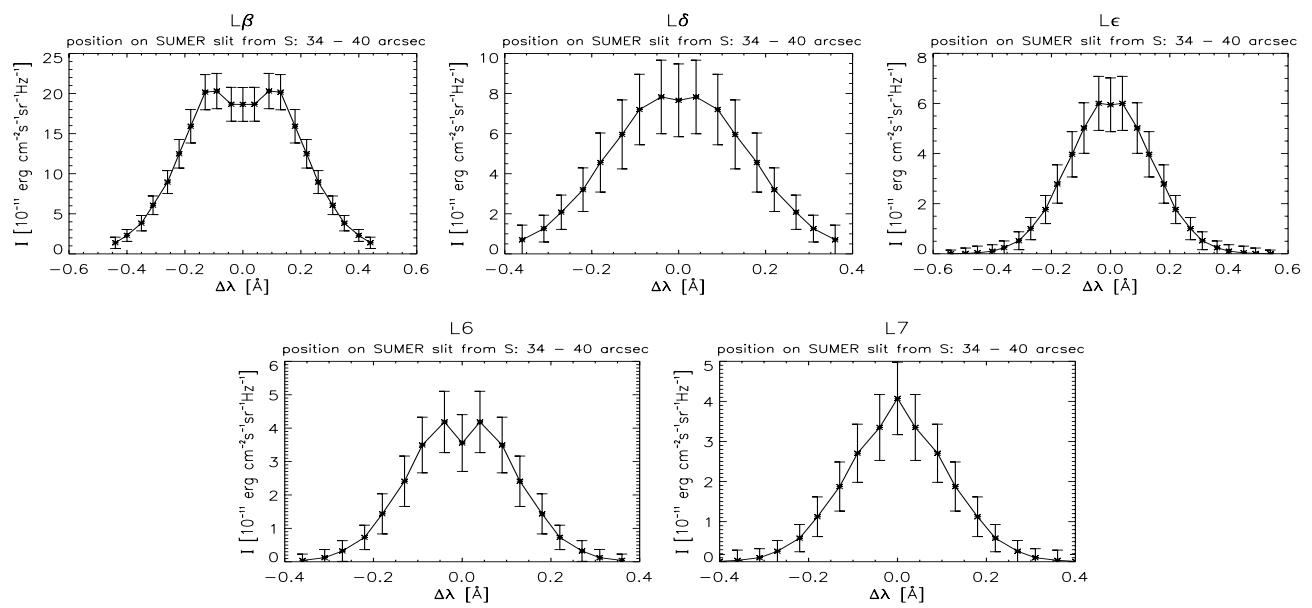

Figure 3. Average profiles of the hydrogen Lyman lines from EUV-extension section I (see Figure 2). The intensity is plotted in units $10^{-11} \mathrm{erg} \mathrm{cm}^{-2} \mathrm{~s}^{-1} \mathrm{sr}^{-1} \mathrm{~Hz}^{-1}$. To smooth the profiles we fitted them with a Gauss function in the wings (as in Stellmacher et al. (2003)). They were also symmetrized to remove the influence of filament plasma flows. For section II we obtained similar profiles.

\section{EUV-extension density and mass estimates}

From the 3D structure previously determined by Schwartz et al. (2004) it was possible to estimate its average density and the mass. We assumed that the EUV-extension contains only hydrogen and helium (abundances of other elements are negligible) and used the common helium abundance 0.1. Then using the definitions for optical thickness at the hydrogen Lyman continuum head and at the $\mathrm{H} \alpha$ line center we computed column mass in each pixel of the CDS raster within the EUV extension (see Heinzel et al. 2003b). Summing the column mass values in all pixels within the EUV-extension area we obtained total mass of the EUV extension. Then the average density is obtained by dividing the total mass by the volume of the EUV extension as determined from its $3 \mathrm{D}$ structure. The average density ranged from $6.3 \times 10^{-16}$ to $4.5 \times 10^{-15} \mathrm{~g} \mathrm{~cm}^{-3}$ and the total mass from $2.4 \times 10^{14}$ to $1.2 \times 10^{15} \mathrm{~g}$ for optical thickness at hydrogen Lyman continuum head between 0.1 and 3 . The resulting total mass is comparable to the mass of the coronal mass ejections (Simnett 2000, Webb 2000).

\section{Estimates of temperature and pressure of EUV-extension plasma}

Using profiles of hydrogen Lyman lines observed by SUMER and the non-LTE filament model of Heinzel et al. (1997) we estimated the temperature and pressure of the EUV-extension plasma. This filament model is represented by horizontal 1D slab irradiated from underneath by the solar atmosphere. There are two prominence-corona transition regions (PCTR) on the top and on the bottom of the $1 \mathrm{D}$ slab distributed symmetrically in the 1D slab. The temperature increases from the center to the edges of the filament steeply in PCTRs but near the center the temperature is almost constant. The pressure is constant in the whole filament. For the geometrical thickness $D$ of the $1 \mathrm{D}$ slab and for height $h$ of the bottom edge of the 1D slab above the solar surface we used values from the 3D structure previously determined by Schwartz et al. (2004). We used values $16000 \mathrm{~km}$ and $20000 \mathrm{~km}$ for $D$ and $h$, respectively, averaged along sections I and II (Figure 2). We computed a grid of such horizontal 1D-slab models for different temperature distributions across $1 \mathrm{D}$ slab and for different pressures. Then we found the best 
model by minimization of $\chi^{2}$ of computed (from horizontal 1D-slab model) and symmetrized observed profiles of hydrogen Lyman lines (Figure 3). For both EUV-extension sections I and II we found that the observed Lyman profiles can be reproduced with:

- low gas pressure $\left(p \approx 10^{-2}{\left.\mathrm{dyn} \mathrm{cm}^{-2}\right)}^{-}\right.$

- temperature in the filament center $2 \times 10^{4}-3 \times 10^{4} \mathrm{~K}$

- temperature at the filament edge around $10^{5} \mathrm{~K}$ (extensive PCTR)

- optical thickness at $\mathrm{H} \alpha$ center much lower than 0.1 (not seen in $\mathrm{H} \alpha$ )

Using these EUV-extension plasma properties and assuming that almost all hydrogen is ionized for such temperatures we calculated average densities $2 \times 10^{-15}-3 \times 10^{-15} \mathrm{~g} \mathrm{~cm}^{-3}$ in extension center and around $6 \times 10^{-16} \mathrm{~g} \mathrm{~cm}^{-3}$ in PCTRs. These values are consistent with values given in Section 3 computed using the $3 \mathrm{D}$ structure only.

\section{Conclusion}

In this paper we presented approximate estimates of the average plasma density and mass of the EUV extension of the filament observed on 15 October 1999. These approximate estimates indicate that the mass of the EUV extension is comparable or even larger than the mass of its counterparts observed in $\mathrm{H} \alpha$. Therefore the large mass loading of CMEs (after filament eruption) could originate from EUV extensions not seen in $\mathrm{H} \alpha$. For more reliable mass and density estimates we need to know the temperature distribution across the horizontal 1D slab and the gas pressure. In this work we estimated temperatures and density using just average Lyman hydrogen line profiles and horizontal 1D-slab non-LTE models for the filament. In future work we plan to construct a finer grid of such models and using optimization techniques we shall derive the spatial distribution of the EUV-filament plasma parameters which will be used for better density and mass estimates.

\section{Acknowledgements}

SoHO is a space mission of international cooperation between ESA and NASA. This work was partly supported by grants A3003203 and S-1003006 of the Grant Agency of the Academy of Sciences of the Czech Republic and the key project K-2043105. P.H., B.S and P.S. are members of the European Solar Magnetism Network (ESMN-HPRNCT-2002-00313) from which this work was partially supported.

\section{References}

Harrison, R.A., Sawyer, E.C., Carter, M.K. et al. 1995 Sol. Phys. 162, 233-290.

Heinzel, P., Anzer, U. \& Schmieder, B. 2003a Sol. Phys. 216, 159-171.

Heinzel, P., Anzer, U., Schmieder, B. \& Schwartz, P. 2003b 'EUV-filaments and their Mass Loading' in Proc. of ISCS 2003 Symposium (A. Wilson ed.), ESA SP-535, 447-457.

Heinzel, P., Schmieder, B. \& Tziotziou, K. 2001 ApJ 561, L223-L227.

Heinzel, P., Schmieder, B. \& Vial, J.-C. 1997 'NLTE ANALYSIS OF SUMER FILAMENT OBSERVATIONS ON SOHO' in Proceedings of Fifth SOHO workshop (A. Wilson ed.), ESA SP-404, 427-432.

Schmieder, B., Lin, Y., Heinzel, P. \& Schwartz, P. 2004 Sol. Phys. 221, 297-323.

Schmieder, B., Tziotziou, K. \& Heinzel, P. 2003 A\&A 401, 361-375.

Schwartz, P., Heinzel, P., Anzer, U. \& Schmieder, B. 2004 A\&A 421, 323-338.

Simnett, G.M. 2000 J. Atm. Sol.-Terr. Phys. 62, 1479-1487.

Stellmacher, G., Wiehr, E. \& Dammasch, I.E. 2003 Sol. Phys. 217, 133-155.

Webb, D.F. 2000 J. Atm. Sol.-Terr. Phys. 62, 1415-1426.

Wilhelm, K., Curdt, W., Marsch, E et al. 1995 Sol. Phys 162, 189-231. 